\title{
Highly Stretchable and Transparent lonogels as Nonvolatile Conductors for Dielectric Elastomer Transducers
}

\section{Citation}

Chen, Baohong, Jing Jing Lu, Can Hui Yang, Jian Hai Yang, Jinxiong Zhou, Yong Mei Chen, and Zhigang Suo. 2014. "Highly Stretchable and Transparent lonogels as Nonvolatile Conductors for Dielectric Elastomer Transducers." ACS Applied Materials \& Interfaces 6 (10) (May 28): 78407845.

\section{Published Version}

doi:10.1021/am501130t

\section{Permanent link}

http://nrs.harvard.edu/urn-3:HUL.InstRepos:13910150

\section{Terms of Use}

This article was downloaded from Harvard University's DASH repository, and is made available under the terms and conditions applicable to Open Access Policy Articles, as set forth at http:// nrs.harvard.edu/urn-3:HUL.InstRepos:dash.current.terms-of-use\#OAP

\section{Share Your Story}

The Harvard community has made this article openly available.

Please share how this access benefits you. Submit a story.

Accessibility 


\title{
Highly Stretchable and Transparent Ionogels as Nonvolatile Conductors for Dielectric Elastomer Transducers
}

\author{
Baohong Chen, ${ }^{1 \#}$ Jing Jing Lu, ${ }^{2 \#}$ Can Hui Yang, ${ }^{1}$ Jian Hai Yang, ${ }^{2}$ Jinxiong Zhou, ${ }^{1}$ Yong Mei \\ Chen, ${ }^{2 *}$ and Zhigang Suo ${ }^{3 *}$
}

${ }^{1}$ State Key Laboratory for Strength and Vibration of Mechanical Structures, International Center for Applied Mechanics and School of Aerospace, Xi'an Jiaotong University, Xi'an 710049, China

${ }^{2}$ Department of Chemistry, School of Science, MOE Key Laboratory for Non-Equilibrium Synthesis and Modulation of Condensed Matter, Xi'an Jiaotong University, Xi'an 710049, China ${ }^{3}$ School of Engineering and Applied Sciences, Kavli Institute of Bionano Science and Technology, Harvard University, Cambridge, Massachusetts 02138, USA

\begin{abstract}
Large deformation of soft materials is harnessed to provide functions in the nascent field of soft machines. This paper describes a new class of systems enabled by highly stretchable, transparent, stable ionogels. We synthesize an ionogel by polymerizing acrylic acid in ionic liquid 1-ethyl-3-methylimidazolium ethylsulfate $\left(\left[\mathrm{C}_{2} \mathrm{mim}\right]\left[\mathrm{EtSO}_{4}\right]\right)$. The ionogel exhibits desired attributes of adequate conductivity $(0.22 \mathrm{~S} / \mathrm{m})$, low elastic modulus $(\sim 3 \mathrm{kPa})$, large rupturing stretch ( 4.6), and negligible hysteresis and degradation after cyclic stretches of large amplitude. Using the ionogel and a dielectric elastomer, we fabricate electromechanical transducers that achieve a voltage-induced areal strain of $140 \%$. The ionogel is somewhat hygroscopic, but the transducers remain stable after a million cycles of excitation in a dry oven and in air. The transparency of the ionogels enable the transducers with conductors placed in the path of light, and the nonvolatility of the ionogels enable the transducers to be used in open air.
\end{abstract}

Keywords: stretchable ionic conductors, transparent transducers, ionic liquids, ionogels, dielectric elastomers 


\section{INTRODUCTION}

Machines in engineering use mostly hard materials, whereas machines in nature are often soft. This conspicuous difference has been an inspiration for the nascent field of soft machines. ${ }^{1-3} \mathrm{~A}$ soft machine uses the large deformation of soft materials to assist humans, ${ }^{4,5}$ operate robots, ${ }^{6-8}$ monitor living tissues, ${ }^{9,10}$ sense environment, ${ }^{11,12}$ shape light, ${ }^{13,14}$ and harvest energy. ${ }^{15}$ A technology under intense development is electromechanical transduction using dielectric elastomers. ${ }^{6,7}$ In such a transduce, a membrane of a dielectric elastomer is sandwiched between two electrical conductors. When a voltage is applied between the conductors, electric charges of the opposite polarities spread on the two faces of the dielectric, causing the dielectric to reduce thickness and increase area.

The conductors on the two faces of the dielectric must be compliant, and be able to deliver voltages up to tens of kilovolts. Making such compliant conductors has been challenging. ${ }^{16}$ Compliant conductors used in dielectric elastomer transducers are traditionally electronic conductors, such as carbon grease, corrugated metal films, carbon nanotubes, graphene sheets, and silver nanowires. ${ }^{16}$ They yield valuable prototypes of devices, but limit applications in several ways. Carbon grease is a viscous liquid, and is opaque and difficult to pattern and integrate in solid-state devices. ${ }^{6}$ Corrugated metal films are also opaque and have relatively small stretchability. ${ }^{17}$ Carbon nanotubes, silver nanowires and graphene sheets exhibit considerable conductivity, but limited stretchability and transmittance. ${ }^{14,18-20}$

Whereas making highly stretchable and transparent electronic conductors has been challenging, a recent work has reported dielectric elastomer transducers using hydrogels as 
stretchable, transparent, ionic conductors. ${ }^{21}$ These transducers can operate at frequencies beyond 10 kilohertz and voltages above 10 kilovolts. The devices are fully transparent to light of all colors, and the electromechanical transduction is achieved without electrochemical reaction. Demonstrated devices include actuators that generate areal strains beyond $100 \%$, and loudspeakers that produce sound over entire audible range. Hydrogels can also function as ionic conductors in devices requiring low voltage and low frequencies, and have been demonstrated as resistive strain sensors. ${ }^{22}$

Hydrogels are easy to make and inexpensive, ideal for demonstrating conceptual designs and for fabricating devices that require biocompatibility. Hydrogels, however, dry out as water evaporates; they are unsuitable for applications in open air. Here we develop nonvolatile, stretchable, transparent conductors: ionogels. An ionogel is a three-dimensional polymer (organic or inorganic) network that hosts an ionic liquid. ${ }^{23}$ Ionic liquids constitute a large and fast growing family of conductive and nonvolatile solvents. ${ }^{24}$ An ionogel combines the attributes of a solid and a liquid: the polymer network provides an elastic solid form, and the ionic liquid enables electrical conduction. Ionogels are being developed intensely for applications in transistors, ${ }^{25}$ batteries, ${ }^{26}$ supercapacitors ${ }^{27,28}$ and fuel cells. ${ }^{23,29}$

Ionogels developed so far, however, do not meet the requirements for being compliant conductors in dielectric elastomer transducers. In such an application, an ionogel must have low elastic modulus and high stretchability, as well as negligible hysteresis and degradation over cyclic deformation. Transducers may operate in dry or moist environment. Other requirements include adhesion between the ionogel and dielectric elastomer, and negligible migration of the ionic liquid into the dielectric elastomer. High transparency and low cost are also desired. The combined requirements post a new and exciting challenge to materials science and engineering. 


\section{FABRICATION OF THE IONOGEL}

We synthesized an ionogel by radical polymerization of acrylic acid (AA) in the ionic liquid 1ethyl-3-methylimidazolium ethylsulfate $\left(\left[\mathrm{C}_{2} \mathrm{mim}\right]\left[\mathrm{EtSO}_{4}\right]\right)$ (Figure 1). In the ionic liquid, we dissolved $\mathrm{AA}$ at $1 \mathrm{~mol} \mathrm{~L}^{-1}$ as the monomer of the polymer network, poly(ethylene glycol) diacrylate (PEGDA) at $0.6 \mathrm{~mol} \%$ of AA as the crosslinker, and $\alpha$-ketoglutaric acid at $1 \mathrm{~mol} \%$ of AA as the initiator. The solution was transferred into a mold made of a pair of glass plates (100 $\mathrm{mm} \times 100 \mathrm{~mm}$ ) separated by a silicone spacer. Then the mold was put into an ultraviolet exposure chamber (SCIENTZ 03-II , Ningbo Scientz Biotechnology Co., LTD). After being irradiated with a power of $50 \mathrm{~W}$ and a wavelength of $365 \mathrm{~nm}$ for $2 \mathrm{~h}$. The solution turned into a stretchable and transparent sheet of poly(acrylic acid) (PAA) ionogel. We controlled the thickness of the ionogel by adjusting the thickness of the silicone spacer.

\section{PROPERTIES OF THE IONOGEL}

We measured the transmittance of the ionogel to light of all colors using a spectrophotometer (UV-2250, SHIMADZU Co.) with a quartz cuvette. A 10-mm thick ionogel showed 96.95\% average transmittance over the visible wavelengths from $380 \mathrm{~nm}$ to $720 \mathrm{~nm}$ (Figure 2a). The inset demonstrates the high transparency of the ionogel by comparing the photos of the ionogel and pure water. Wereas water is a liquid and flows, the ionogel is a solid and retains the shape when we placed the container upside down.

We studied the mechanical behavior of the ionogel using a tensile-machine (CMT6503, MTS). The ionogel had Young's modulus $3.1 \pm 0.6 \mathrm{kPa}$, fracture strength $7.4 \pm 0.4 \mathrm{kPa}$, and fracture stretch $4.6 \pm 0.3$. We further measured the cyclic stretch-stress curves of the ionogel by using rectangular specimens of dimensions $4.5 \mathrm{~mm} \times 7 \mathrm{~mm} \times 0.5 \mathrm{~mm}$ and a dynamic mechanical 
analysis tester (DMA Q800). The ionogel exhibited relative small hysteresis after hundreds of cycles of loading and unloading (Figure 2b). Young's modulus of the ionogel decreased in initial cycles, and became stable after about 150 cycles. Overall, the ionogel exhibited good mechanical reversibility.

We compared the volatility of the ionic liquid, the ionogel and a hydrogel by weighing samples placed in a thermostatic chamber held at $100{ }^{\circ} \mathrm{C}$. The weight of the ionic liquid decreased $4.23 \%$ and became stable within $12 \mathrm{~h}$. The weight of ionogel decreased $12.43 \%$ and became stable within $12 \mathrm{~h}$. The ionnic liquid and the ionogel were somewhat hygrospcoic, and the initial loss of weight was due to the evaporation of the dissolved water. By contrast, the weight of a poly(acrylic acid) hydrogel contained a large qunatity of water, and its weight dropped steeply and decreased $93.69 \%$ within $1 \mathrm{~h}$ as water evaporated. The photos compare the ionogel and the hydrogel after being in the thermostatic chamber for $12 \mathrm{~h}$ (Figure 2c, inset).

We measured the conductivity of the ionic liquid by using a conductivity meter (DDSJ308A), and measured the conductivity of the ionogel by using an impedance analyzer (Agilent E4980A). In both cases, the measurements were carried out at room temperature $\left(\sim 25^{\circ} \mathrm{C}\right)$. Discs of the ionogel, $12.0 \mathrm{~mm}$ in diameter and $2.0 \mathrm{~mm}$ in thickness, were sandwiched between two mirror-finished cooper electrodes. We applied two levels of voltage, $10 \mathrm{mV}$ and $1 \mathrm{~V}$, and found that the measured conductivities were essentially independent of the voltage in this range. To eliminate the effect of water dissolved in the ionic liquid and ionogel, before the conductivity was measured, both of them were held in the thermostatic chamber at $100{ }^{0} \mathrm{C}$ for $10 \mathrm{~h}$. Afterwards, the measured conductivity of ionic liquid was $0.23 \mathrm{~S} \mathrm{~m}^{-1}$, which is somewhat lower than the value reported in the literature $\left(0.38 \mathrm{~S} \mathrm{~m}^{-1}\right){ }^{30}$ The conductivity of the ionogel was $0.22 \pm 0.01 \mathrm{~S} \mathrm{~m}^{-1}$. To place these values in context, recall that the conductivities of salt- 
containing hydrogels are $\sim 10^{2} \mathrm{~S} \mathrm{~m}^{-1},{ }^{21,33}$ and the conductivities of many ionic liquids are $\sim 1$ $\mathrm{S} \mathrm{m}^{-1} \cdot{ }^{30}$ This low conductivity of $1 \mathrm{~S} \mathrm{~m}^{-1}$ is comparable to that of carbon grease, ${ }^{31}$ an electrical conductor commonly used for dielectric elastomer transducers. Our ionogel is somewhat hygroscopic. When the ionogel absorbs water, ions become more mobile, and the electrical conductivity increases (Table 1).

We also measured the electrical resistance of a dumbbell-shaped ionogel when stretched by uniaxial forces using the four-point method (Figure 2d). This method measures electrical resistance of a conductor using two external probes and two inner probes. The two external probes are connected to the ends of the conductor, and to the power source and the galvanometer to supply and measure electrical current. The two inner probes are connected to two points in the central uniform part of the conductor, and to a voltmeter to measure the voltage. The ratio of the voltage to the electrical current determines the electrical resistance between the two inner probes. Also plotted in Figure 2d is the theoretical prediction of an ideal conductor, for which the resistivity and volume are independent of stretch. When the length of the ideal conductor is stretched by a factor of $\lambda$, the cross-sectional area of the conductor reduces by a factor of $\lambda$, so that the ratio of the resistance of the stretched conductor $(R)$ to that of the undeformed conductor $\left(R_{0}\right)$ is $R / R_{0}=\lambda^{2}$. The resistivity of the ionogel increased somewhat when the ionogel was stretched; however, the deviation of the measured resistance from the prediction of the ideal conductor is relatively small.

We further measured the change in the electrical resistance when the ionogel is subject to cyclic loading and unloading between stretches 1 and 4 . The increase of the electrical resistance of the ionogel was modest after hundreds of cycles (Figure 2d, inset). This result further 
confirms that the ionogel suffers negligible degradation after cyclic stretches of large amplitude. The small change in resistance of the ionogel may be compared with that of other conductors under cyclic loads. For example, the electrical resistance of nanotube films increased 100-1000 times after hundreds of cycles between stretches 1 and 2. ${ }^{18}$

When a sheet of the ionogel is in contact with a membrane of a dielectric elastomer, the ionic liquid in the ionogel may migrate into the dielectric elastomer, causing premature failure. We immersed the membrane of a dielectric elastomer (VHB 4910,3M), thickness $1 \mathrm{~mm}$, into a large amount of the ionic liquid, and after wiped by filter paper, measured the weight of the dielectric elastomer several times over one month. The weight of VHB did not change. This indicates that $\mathrm{VHB}$ is inert to the $\left[\mathrm{C}_{2} \mathrm{mim}\right]\left[\mathrm{EtSO}_{4}\right]$ ionic liquid.

\section{ACTUATOR MADE BY LAMINATING IONOGEL AND DIELECTRIC ELASTOMER}

We fabricated an actuator using the ionogel as the conductors and the VHB as the dielectric elastomer (Figure 3a). The VHB was stretched radially to three times its original radius, and fixed between two circular rigid plastic frames of inner diameter of $66.5 \mathrm{~mm}$. Sheets of $0.2-\mathrm{mm}$ thick ionogel were cut into a circular shape of 17.0-mm diameter using a laser cutting system (Versa Laser VLS2.30, Universal Laser Systems). Two circular-shaped sheets of the ionogel were then gently placed on the two faces of the VHB. Each sheet of the ionogel was linked, through a thin line of the ionogel, to an aluminum electrode placed on the rigid frame. The two aluminum electrodes were connected to a power source (Model 30/20, TREK). The VHB has been marketed as an adhesive tape. The adhesion between the VHB and the ionogel was adequate; we did not observe delamination during actuation.

This design places the electrode (electronic conductor), electrolyte (ionic conductor) and 
dielectric (insulator) in series (Figure 3b). ${ }^{21}$ For some combinations of the electrode and electrolyte, if the voltage across the electrode/electrolyte interface is within a certain range $(\sim 1$ V), electrons and ions do not cross the interface, no electrochemical reaction occurs, and the interface forms an electrical double layer, like a capacitor. ${ }^{21}$ Charges in the electrode and in the electrolyte are separated over nanometers. By contrast, charges on the two faces of the dielectric elastomer are separated by its thickness (on the order of $0.1 \mathrm{~mm}$ in a stretched dielectric). Consequently, the electrical double layer has an enormous capacitance compared to the dielectric elastomer. When a high voltage is applied between the two aluminum electrodes, the voltage across the electrical double layer is much smaller than $1 \mathrm{~V}$, and nearly all the applied voltage drops across the dielectric elastomer. In our experiments, the permittivity of dielectric elastomer is $\varepsilon_{\mathrm{DE}} \sim 10^{-11} \mathrm{~F} \mathrm{~m}^{-1}$, the thickness of dielectric is $H_{D E} \sim 10^{-4} \mathrm{~m}$, the capacitance per unit area of the electrical double layer is $c_{E D L} \sim 10^{-1} \mathrm{~F} \mathrm{~m}^{-121}$ and the areal ratio between dielectric and electrical double layer is $A_{D E} / A_{E D L} \sim 10^{2}$. When a voltage is applied between the two metallic electrodes, the voltage across the electrical double layer $V_{E D L}$ relates to the voltage across the dielectric $V_{D E}$ as ${ }^{21}$

$$
\frac{V_{E D L}}{V_{D E}}=\frac{A_{D E}}{A_{E D L}} \frac{\varepsilon_{D E}}{H_{D E}} \frac{1}{c_{E D L}}
$$

Using the representative numbers listed above, we find that $V_{E D L} / V_{D E} \sim 10^{-4}$. Consequently, when the voltage across dielectric before breakdown is $4 \mathrm{kV}$, the voltage across the electrical double layer is just $0.4 \mathrm{~V}$, which is lower than the decomposition voltage of the ionic liquid. A small voltage across the electrical double layer prevents electrochemical reaction, and a large voltage across the dielectric elastomer enables electromechanical transduction. 
When the voltage is applied, the charges of the opposite polarities on the two faces of the dielectric elastomer attract, so that the actuator reduces thickness and expands area (Figure 3c, 3d). The elastic modulus of the poly(acrylic acid) ionogel is a few $\mathrm{kPa}$, but that of the dielectric is on the order of $100 \mathrm{kPa}$. Consequently, the soft ionogel negligibly constrains the actuation. When the voltage was ramped up at a $10 \mathrm{~V} \mathrm{~s}^{-1}$, the areal strains were recorded until the dielectric elastomers failed by electrical breakdown. The actuator achieved an areal strain of $140 \%$. The stretch-voltage curve of the actuator using the ionogel was similar to the actuator using carbon grease (Figure 3e). The experimental data matched closely with the predictions using an existing theoretical model. ${ }^{32}$ After the actuator made with the ionogel and dielectric elastomer was placed in the open air for several weeks, we could still obtain the same large areal strain.

The electromechanical response is frequency-dependent. Subject to cyclic voltage, the actuator oscillated and, due to viscoelasticity of the VHB, the amount of actuation drifted in the initial cycles. ${ }^{33}$ After several cycles, the actuator oscillated steadily. We plot the steady areal strain (maximum strain minus minimum strain in one cycle) as a function of the frequency (Figure 3f).

The frequency of actuation is limited by mechanical resonance, not by electrical resistance. The time delay in charging a capacitor, i.e. the RC delay, scales as ${ }^{21}$

$$
\tau_{R C} \sim \mathrm{c}_{D E} A_{D E} R
$$

In our experiment, the capacitance of dielectric elastomer per unit area is $\mathrm{c}_{D E}=10^{-8} \mathrm{~F} \mathrm{~m}^{-2}$, the area of dielectric elastomer is $A_{D E}=10^{-2} \mathrm{~m}^{2}$ and the sheet resistance of ionogel is $R=10^{4} \mathrm{ohm}$ square $^{-1}$, so the RC delay of the actuator is $\tau_{R C} \sim 10^{-6} \mathrm{~s}$. The mechanical resonance sets another time scale ${ }^{21}$ 


$$
\tau_{\text {inertia }} \sim \sqrt{A_{D E} \rho / Y}
$$

where $\rho$ is the mass density and $Y$ is the elastic modulus. For representative values $A_{D E}=10^{-2} \mathrm{~m}^{2}, \rho=10^{3} \mathrm{~kg} \mathrm{~m}^{-3}$ and $Y=10^{6} \mathrm{~N} \mathrm{~m}^{-2}, \tau_{\text {inertia }} \sim 10^{-3} \mathrm{~s}$, corresponding to a frequency of $1 \mathrm{kHz}$. The areal strain of the actuator using ionogel (Figure 3f) is comparable to the areal strain of the actuator using ionic liquid (Figure S6 of Ref.21). In both cases, the areal strain reduces rapidly with the frequency of excitation. The reason for this rapid reduction is unclear. More systematic experiments and calculations are needed.

The ionogel is somewhat hygroscopic, but the absorption of water by the ionogel does not negatively impact the performance of the actuators. We carried out experiments to compare the performance of actuators in an oven and in air. A total of six actuators were prepared. Three of them were placed in the oven of relative humidity $10 \pm 0.4 \%$, and the others were placed in air of relative humidity $54 \pm 5 \%$. The temperature in the oven and in air was $20 \pm 1{ }^{\circ} \mathrm{C}$. We applied a sinusoidal voltage of amplitude $3300 \mathrm{~V}$ and frequency $20 \mathrm{~Hz}$. The areal strain of actuators was recorded after every 100 thousands cycles and the experiment was terminated after $1000 \mathrm{k}$ cycles. When we measured the areal strain, the frequency of the applied voltage was turned down to $1 / 16 \mathrm{~Hz}$ for observing a relative large and clearly visible areal strain. After the measurement, the frequency of voltage was turned back to $20 \mathrm{~Hz}$ for more cycles. The difference in the areal strain of the actuators tested in the oven and in air was small (Figure 4). In both environments, the actuators could work for at least a million cycles. 


\section{CONCLUSION}

In summary, we have synthesized a highly stretchable, transparent, stable ionogel, and demonstrated its use as a nonvolatile, compliant ionic conductor for dielectric elastomer transducers. The transparency of the ionogel will enable devices with conductors placed in the path of light, such as tunable lenses, ${ }^{14}$ transparent loudspeakers, ${ }^{21}$ and active-noise-cancellation windows. $^{34}$ The nonvolatility of the ionogel will enable transducers to be used in open air. Ionic liquids constitute a large family of conductive solvents, so that ionogels with various attributes can be synthesized to broaden the applications of highly deformable electromechanical transducers. 
(a)<smiles>C=CC(=O)OCCC(C)OC(=O)C=C</smiles>

AA PEGDA

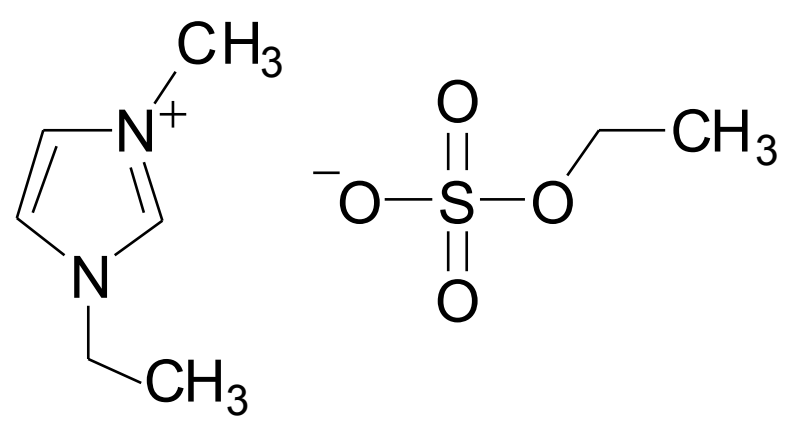

$\left[\mathrm{C}_{2} \mathrm{mim}\right]\left[\mathrm{EtSO}_{4}\right]$

(b)

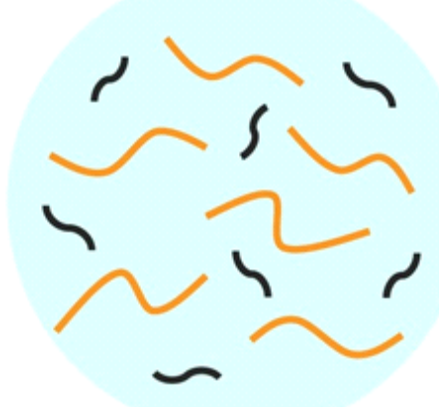

Solution

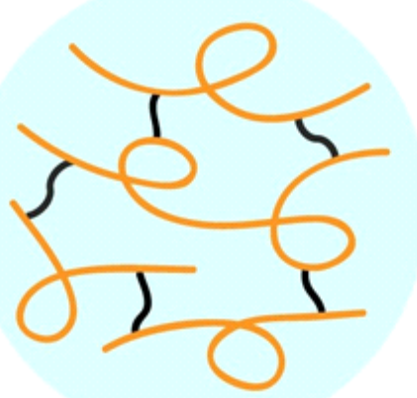

lonogel

\section{: Acrylic acid : Ionic liquid $\sim$ :Crosslinker}

Figure 1. Preparation of the poly(acrylic acid) ionogel. (a) Ingredients: acrylic acid (AA) as the precursor of the polymer network, poly(ethylene glycol) diacrylate (PEGDA) as the crosslinker, and 1-ethyl-3-methylimidazolium ethylsulfate $\left(\left[\mathrm{C}_{2} \mathrm{mim}\right]\left[\mathrm{EtSO}_{4}\right]\right)$ as the ionic liquid. (b) The ingredients are mixed and stirred for 20 minutes, forming a homogeneous solution. The solution forms an ionogel after exposing to the UV light for 2 hours. 

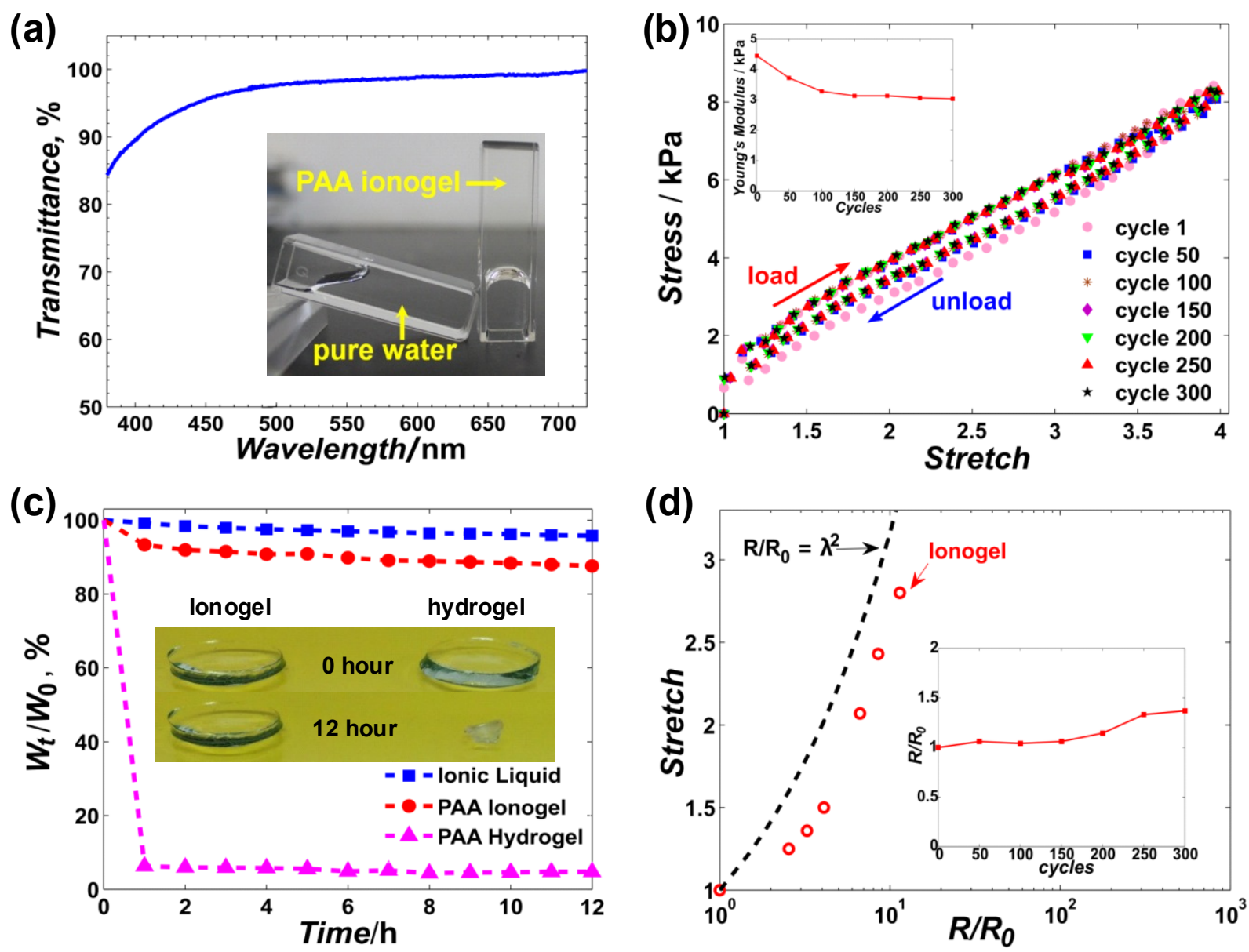

Figure 2. Properties of the poly(acrylic acid) ionogel. (a) Transmittance of a 10-mm thick ionogel to visible light of all colors. Inset: photos of pure water and the ionogel. (b) Stressstretch curves of the ionogel subject to tensile load and unload. Inset: the change of Young's modulus with the increasing number of cyclic stretch. (c) Change of weight of the ionic liquid, ionogel and hydrogel in a thermostatic chamber at $100^{\circ} \mathrm{C}$. Inset: photos of the ionogel and hydrogel before and after being in the thermostatic chamber for 12 hours. (d) The change of electrical resistance as the ionogel is stretched. Inset: the change of electrical resistance of the ionogel after being cyclically stretched. 
(a)

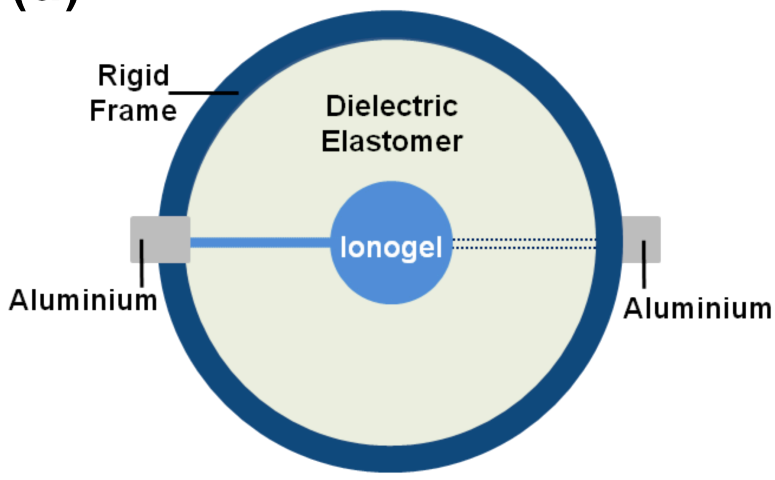

(c)

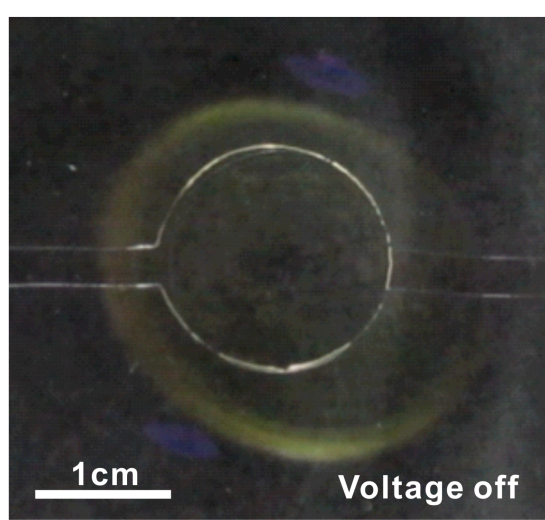

$(\mathbf{e})$

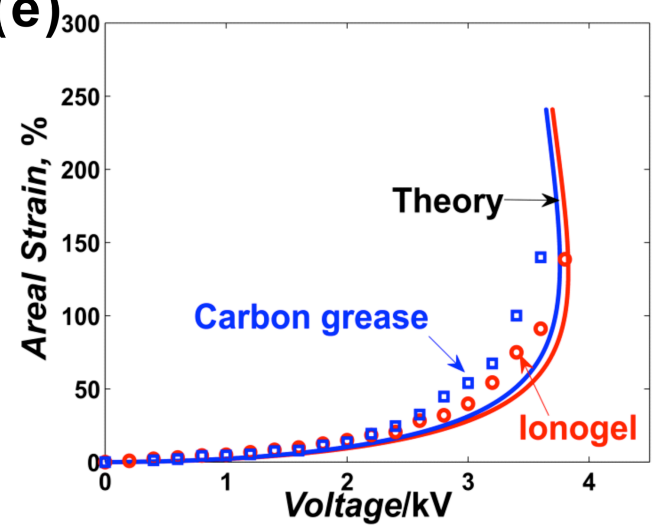

(b)

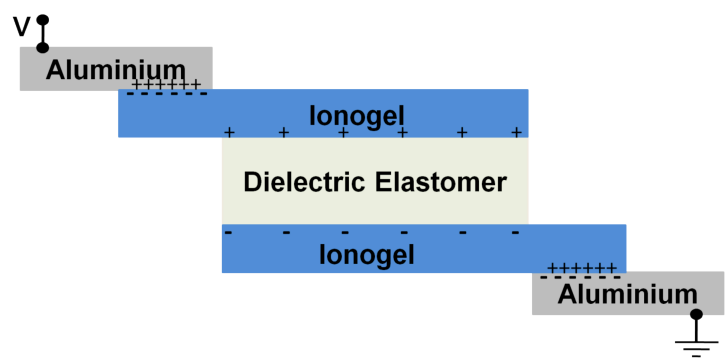

(d)

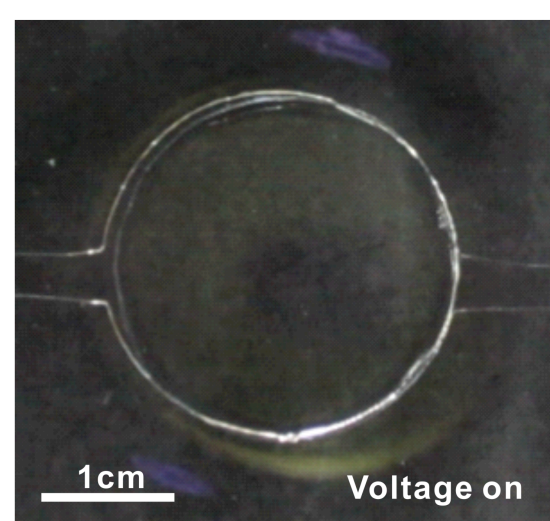

(f)

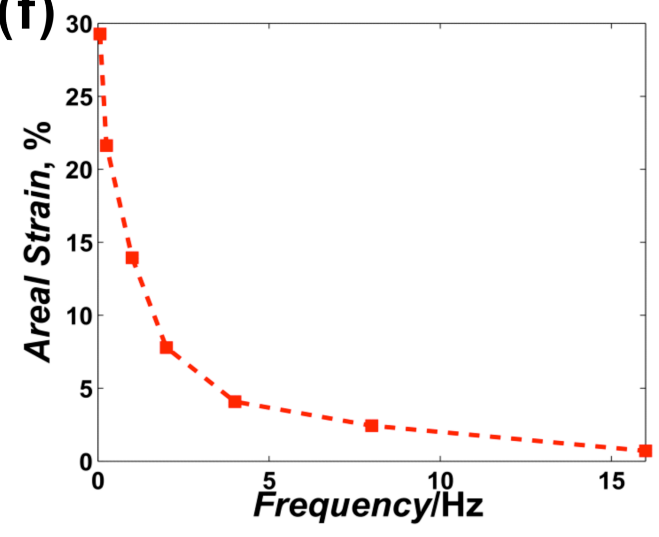

Figure 3. An actuator made by layering the poly(acrylic acid) ionogel with a dielectric elastomer. (a) A membrane of the dielectric elastomer is stretched and fixed between two rigid plastic frames. Each face of the dielectric membrane is attached with a layer of the ionogel. Thin lines of the ionogel extend to the rigid frame and meet aluminium wires, which are connected to 
the power source. (b) The side view of the actuator. (c) When the voltage is off, the actuator is in the as-prepared state. (d) When the two aluminum wires are subject to a voltage, the actuator expands in area. (e) The areal strain as a function of voltage for actuators using carbon grease and ionogel as conductors. (f) The areal strain of an actuator made of the dielectric elastomer and the ionogel changes with the frequency of excitation increases.

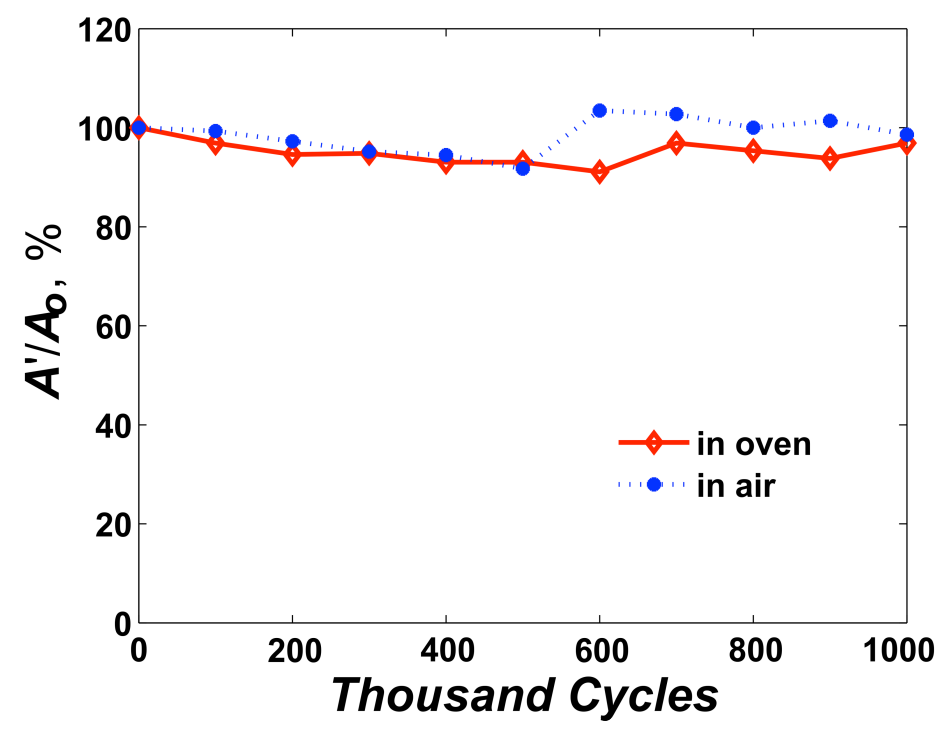

Figure 4. The change of the areal strain of actuators after cycles of excitation in oven of relative humidity $10 \pm 0.4 \%$ and in air of relative humidity $54 \pm 5 \%$. $A^{\prime}$ is the areal strain measured after a certain number of cycles, and $A_{0}$ is the areal strain measured in the first cycle. 
Table 1. The conductivities of ionic conductors.

\begin{tabular}{|c|c|c|c|c|c|c|}
\hline Samples & $\begin{array}{c}\mathrm{IL} *(10 \mathrm{~g}) \\
\text { Dried }\end{array}$ & $\begin{array}{l}\text { IL }(10 \mathrm{~g}) \\
\text { In air }\end{array}$ & $\begin{array}{l}\mathrm{IL}(10 \mathrm{~g}) \\
+\mathrm{H}_{2} \mathrm{O} \\
(1.58 \mathrm{~g})\end{array}$ & $\begin{array}{c}\mathrm{IL}(10 \mathrm{~g}) \\
+\mathrm{AA}^{* *}(0.5765 \mathrm{~g}) \\
+\mathrm{H}_{2} \mathrm{O}(1.672 \mathrm{~g})\end{array}$ & $\begin{array}{c}\text { Ionogel } \\
\text { Dried }\end{array}$ & $\begin{array}{l}\text { Ionogel } \\
\text { In air }\end{array}$ \\
\hline $\begin{array}{l}\text { Conductivity } \\
\left(\mathrm{S} \mathrm{m}^{-1}\right)^{* * *}\end{array}$ & 0.23 & 0.42 & 1.55 & 1.53 & 0.22 & 1.43 \\
\hline
\end{tabular}

*IL represents ionic liquid $\left[\mathrm{C}_{2} \mathrm{mim}\right]\left[\mathrm{EtSO}_{4}\right]$. Dried means the water in samples was removed by heating in thermostatic chamber before measurement.

**AA is the monomer of poly(acrylic acid), which constructs the network of the poly(acrylic acid) ionogel. $* * *$ Samples were measured under the room temperature $\left(\sim 25{ }^{0} \mathrm{C}\right)$.

\section{Authors Information}

\section{Corresponding Authors}

* E-mail: chenym@mail.xjtu.edu.cn

* E-mail:suo@seas.harvard.edu

\section{Notes}

The authors declare no competing financial interest.

\section{Authors Contribution}

${ }^{\#}$ These authors contributed equally.

\section{Acknowledgements}

This research is supported by Natural Science Foundation of China (Grants 51173144, 51073127, 11072185, 11372239 and 11321062), the Research Fund for the Doctoral Program of Higher Education of China (Grant 201110040), Scientific Research Foundation for the Returned

Overseas Chinese Scholars, State Education Ministry, the International Science \& Technology Cooperation Program Supported by Ministry of Science and Technology of China (2013836-16) 
and Shaanxi Province (2013KW14-02), the Key Innovational Research Team Program Supported by the Shaanxi Province (2013KCT-05), Fundamental Research Funds for the Central Universities. ZS acknowledges the support of NSF MRSEC (DMR-0820484) and visiting appointment at the International Center for Applied Mechanics.

\section{References}

(1) Calvert, P. Hydrogels for Soft Machines. Adv. Mater. (Weinheim, Ger.) 2009, 21, 743756.

(2) Suo, Z. Mechanics of stretchable electronics and soft machines. MRS Bull. 2012, 37, 218225.

(3) Morin, S. A.; Shepherd, R. F.; Kwok, S. W.; Stokes, A. A.; Nemiroski, A.; Whitesides, G. M. Camouflage and Display for Soft Machines. Science 2012, 337, 828-832.

(4) Niu, X.; Yang, X., Brochu, P.; Stoyanov, H.; Yun, S.; Yu, Z.; Pei, Q. Bistable LargeStrain Actuation of Interpenetrating Polymer Networks. Adv. Mater. (Weinheim, Ger.) 2012, 24, 246513-6519.

(5) Roche, E. T.; Wohlfarth, R.; Overvelde, J. T. B.; Vasilyev, N. V.; Pigula, F. A.; Mooney, D. J.; Bertoldi, K.; Walsh, C. J. A Bioinspired Soft Actuated Material. Adv. Mater. (Weinheim, Ger.) 2014, 26, 1200-1206.

(6) Pelrine, R.; Kornbluh, R.;. Pei, Q; Joseph, J. High-Speed Electrically Actuated Elastomers with Strain Greater Than 100\%. Science 2000, 287, 836-839. 
(7) Anderson, I. A.; Gisby, T. A.; McKay, T. G.; OBrien, B. M.; Calius, E. P. Multifunctional dielectric elastomer artificial muscles for soft and smart machines. J. Appl. Phys. (Melville, NY, U. S.) 2012, 112, 041101.

(8) Chossat, J. -B.; Park, Y.-L; Wood, R. J.; Duchaine, V. A Soft Strain Sensor Based on Ionic and Metal Liquids. IEEE Sens. J. 2013, 13, 3405-3414.

(9) Graudejus, O.; Yu, Z.; Jones, J.; Morrison, B.; Wagner, S. Characterization of an Elastically Stretchable Microelectrode Array and Its Application to Neural Field Potential Recordings. J. Electrochem. Soc. 2009, 156, 85-94.

(10) Kim, D.-H.; Lu, N.; Ma, R.; Kim, Y.-S.; Kim, R.-H.; Wang, S.; Wu, J.; Won, S. M.; Tao, H.; Islam, A.; Yu, K. J.; Kim, T.; Chowdhury, R.; Ying, M.; Xu, L.; Li, M.; Chung, H.J.; Keum, H.; McCormick, M.; Liu, P.; Zhang, Y.-W.; Omenetto, F. G.; Huang, Y.; Coleman, T.; Rogers, J. A. Epidermal Electronics. Science 2011, 333, 838-843.

(11) Hammock, M. L.; Chortos, A.; Tee, B. C. K.; Tok, J. B. H.; Bao, Z. 25th Anniversary Article: The Evolution of Electronic Skin (E-Skin): A Brief History, Design Considerations, and Recent Progress. Adv. Mater. (Weinheim, Ger.) 2013, 25, 5997-6038.

(12) Kaltenbrunner, M.; Sekitani, T.; Reeder, J.; Yokota, T.; Kuribara, K.; Tokuhara, T.; Drack, M.; Schwödiauer, R.; Graz, I.; Bauer-Gogonea, S.; Bauer, S.; Someya, T. An ultra-lightweight design for imperceptible plastic electronics. Nature 2013, 499, 458-463.

(13) Carpi, F.; Frediani, G.; Turco, S.; Rossi, D. D. Bioinspired Tunable Lens with MuscleLike Electroactive Elastomers. Adv. Funct. Mater. 2011, 21, 4152-4158.

(14) Shian, S.; Diebold, R. M.; Clarke, D. R. Tunable lenses using transparent dielectric elastomer actuators. Opt. Express 2013, 21, 8669-8676. 
(15) Kornbluh, R. D.; Pelrine, R.; Prahlad, H.; Wong-Foy, A.; McCoy, B.; Kim, S.; Eckerle, J.; Low, T. Dielectric elastomers: Stretching the capabilities of energy harvesting. MRS Bull. 2012, 37, 246-253.

(16) Rosset, S.; Shea, H. R. Flexible and stretchable electrodes for dielectric elastomer actuators. Appl. Phys. A: Mater. Sci. Process. 2012, 110, 281-308.

(17) Benslimane, M.; Gravesen, P.; Sommer-Larsen, P. Mechanical properties of Dielectric Elastomer Actuators with smart metallic compliant electrodes. Proc. SPIE 2002, 4695, $150-157$.

(18) Hu, L.; Yuan, W.; Brochu, P.; Gruner, G.; Pei, Q. Highly stretchable, conductive, and transparent nanotube thin films. Appl. Phys. Lett. 2009, 94, 161108

(19) Scardaci, V.; Coull, R.; Coleman, J. N. Very thin transparent, conductive carbon nanotube films on flexible substrates. Appl. Phys. Lett. 2010, 97, 023114

(20) Zang, J.; Ryu, S.; Pugno, N.; Wang, Q.; Tu, Q.; Buehler, M. J.; Zhao, X. Multifunctionality and control of the crumpling and unfolding of large-area graphene. Nat. Mater. 2013, 12, 321-325.

(21) Keplinger, C.; Sun, J.-Y.; Foo, C. C.; Rothemund, P.; Whitesides, G. M.; Suo, Z. Stretchable, Transparent, Ionic Conductors. Science 2013, 341, 984-987.

(22) Manandhar, P.; Calvert, P. D.; Buck, J. R. Elastomeric Ionic Hydrogel Sensor for Large Strains. IEEE Sens. J. 2012, 12, 2052-2061.

(23) Bideau, J. L.; Viau, L.; Vioux, A. Ionogels, ionic liquid based hybrid materials. Chem. Soc. Rev. 2011, 40, 907-925.

(24) Werner, S.; Haumann, M.; Wasserscheid, P. Ionic Liquids in Chemical Engineering. Annu. Rev. Chem. Biomol. Eng. 2010, 1, 203-230. 
(25) Cho, J. H.; Lee, J.; He, Y.; Kim, B.; Lodge, T. P.; Frisbie, C. D. High-Capacitance Ion Gel Gate Dielectrics with Faster Polarization Response Times for Organic Thin Film Transistors. Adv. Mater. (Weinheim, Ger.) 2008, 20, 686-690.

(26) Bideau, J. L.; Ducros, J.-B.; Soudan, P.; Guyomard, D. Solid-State Electrode Materials with Ionic-Liquid Properties for Energy Storage: the Lithium Solid-State Ionic-Liquid Concept. Adv. Funct. Mater. 2011, 21, 4073-4078.

(27) Balducci, A.; Bardi, U.; Caporali, S.; Mastragostino, M.; Soavi, F. Ionic liquids for hybrid supercapacitors. Electrochem. Commun. 2004, 6, 566-570.

(28) Saricilar, S.; Antiohos, D.; Shu, K.; Whitten, P. G.; Wagner, K.; Wang, C.; Wallace, G. G. High strain stretchable solid electrolytes. Electrochem. Commun. 2013, 32, 47-50.

(29) Kubo, W.; Kitamura, T.; Hanabusa, K.; Wada, Y.; Yanagida, S. Quasi-solid-state dyesensitized solar cells using room temperature molten salts and a low molecular weight gelator. Chem. Commun. (Cambridge, U. K.) 2002, 4, 374-375.

(30) Patel, D. D.; Lee, J. M. Applications of Ionic Liquids. Chem. Rec. 2012, 12, 329-355.

(31) http://www.mgchemicals.com/products/greases-and-lubricants/conductivegreases/carbon-conductive-grease-846/

(32) Bozlar, M.; Punckt, C.; Korkut, S.; Zhu, J.; Foo, C. C.; Suo, Z.; Aksay, I. A. Dielectric elastomer actuators with elastomeric electrodes. Appl. Phys. Lett. 2012, 101, 091907.

(33) Bai, Y.; Jiang, Y.; Chen, B.; Foo, C. C.; Zhou, Y.; Xiang, F.; Zhou, J.; Wang, H.; Suo, Z. Cyclic performance of viscoelastic dielectric elastomers with solid hydrogel electrodes. Appl. Phys. Lett. 2014, 104, 062902.

(34) Yu, X.; Rajamani, R.; Stelson, K.; Cui, T. Carbon nanotube-based transparent thin film acoustic actuators and sensors. Sens. Actuators, A 2006, 132, 626-631. 
Figure for Table of Content

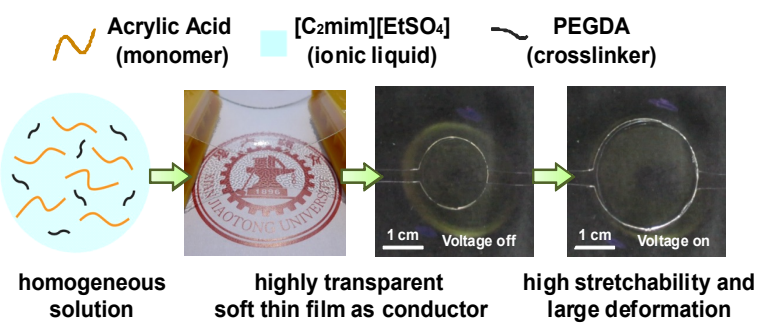

